\title{
EVOLUCIÓN DE LA POBLACIÓN ACTIVA EN LOS MUNICIPIOS DEL INTERIOR MONTAÑOSO DE LA MARINA
}

\author{
M. J. Santos Deltell
}

\begin{abstract}
RESUMEN
El cambio de la población que se produce en los pueblos de la Montaña de Villajoyosa se debe a varios factores: Cambio de las actividades económicas, los campesinos dejan de serlo para trabajar en la industria o para formar parte de la población activa del sector terciario; otra de las causas atribuidas a este cambio sería el envejecimiento de la población y la emigración continuada en los últimos treinta años.

RÉSUMÉ

Le changement de la population qui s'est produit dans les villages de la montagne de Villajoyosa est dû à plusieurs facteurs: changements des activités économiques, les paysans passent à être des travailleurs dans l'industrie, ou à former partie de la population active du secteur terciaire; une autre cause serait le vieillissement de toute la population et l'emigration continué pendant les trente dernières anneés.
\end{abstract}

\section{Introducción}

Esta zona comprende los municipios que se asientan en las laderas montañosas de la Aitana, el Puig Campana y el Valle de Guadalest.

Se trata de núcleos que han seguido una misma trayectoria histórica y demográfica. Antiguamente dependían de la villa de Guadalest, y hoy en día, desgajados de la Baronía a la que pertenecían, muestran unos rasgos comunes: pérdida de población en todos ellos, desde 1857 hasta 1981, superior a veces al 30\%, envejecimiento demográfico, la mayor parte de sus habitantes son mayores de 40 años, y que el censo de 1981 refleja valores absolutos de población en torno a los 1.000 vecinos.

Son, Guadalest, Castell de Castells y Confrides. Fuera de este valle también vamos a estudiar otros dos ejemplos que estimamos convenientes: los municipios de Polop y Relleu.

Para realizar este trabajo se ha contado con la fuente de información que proporcionan los censos o padrones municipales de habitantes.

Los pueblos analizados debían contar con el contrapunto que supone analizar los datos de 1950 y los de 1981, para ver la evolución que se había operado en estos treinta años. 


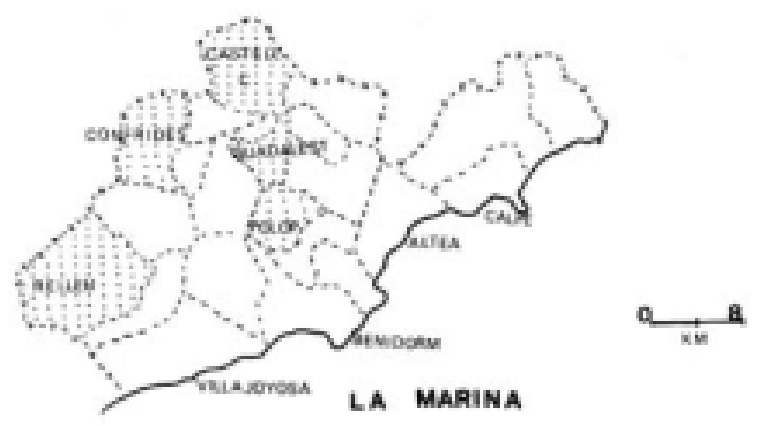

Figura 1.

Por regla general, se pueden observar los mismos factores que son comunes en esta serie de municipios.

En primer lugar destaca el progresivo abandono de los activos que se dedican al sector primario, en beneficio de otros sectores de actividad, como puedan ser el terciario o la industria (rama de la construcción). Las actividades fabriles no están muy desarrolladas, sino que se trata de incipientes establecimientos o talleres de pequeña importancia. Es frecuente encontrar talleres clandestinos de industria sumergida (a través de la encuesta directa), actividad ésta bastante extendida en todos esos lugares del interior montañoso de la provincia de Alicante. Estos núcleos que vivían volcados en una agricultura pobre de secano, con escasos ingresos, tuvieron que buscar otra solución a lo perentorio de su economía. Este resultado vino aparejado con la práctica del trabajo a domicilio, es decir, en algunos de estos pueblos se reparte el trabajo una vez a la semana y se recoge después de realizado, redistribuyéndose a continuación a las empresas que lo habían encargado. Aparece el caso de Relleu, donde de un tiempo a esta parte se cosen pantalones de caballero. Una vez por semana el trabajo se envía a los centros de San Vicente del Raspeig, Alicante y Alcoy, que se encargan de comercializarlos.

Se trata de una actividad eminentemente femenina, que registra entre otros problemas: el abuso y la explotación que sufren estas mujeres, ya que por piezas realizadas se vienen a pagar unas 300 pesetas (datos de 1988).

Si se sigue llevando a cabo, es por ser considerado entre ellas como una fuente de ingresos más, complementaria de las aportaciones familiares que consigue el marido y porque se puede realizar en la propia casa en horario flexible.

Es un trabajo que absorbe mayoritariamente a las mujeres casadas, ya que las solteras suelen hacerlo en la industria o en los servicios (generalmente se emplean hasta que se casan y después vienen a realizar el trabajo a domicilio que les es más cómodo).

No existen fuentes oficiales que registren este tipo de actividad denominada falsamen- 


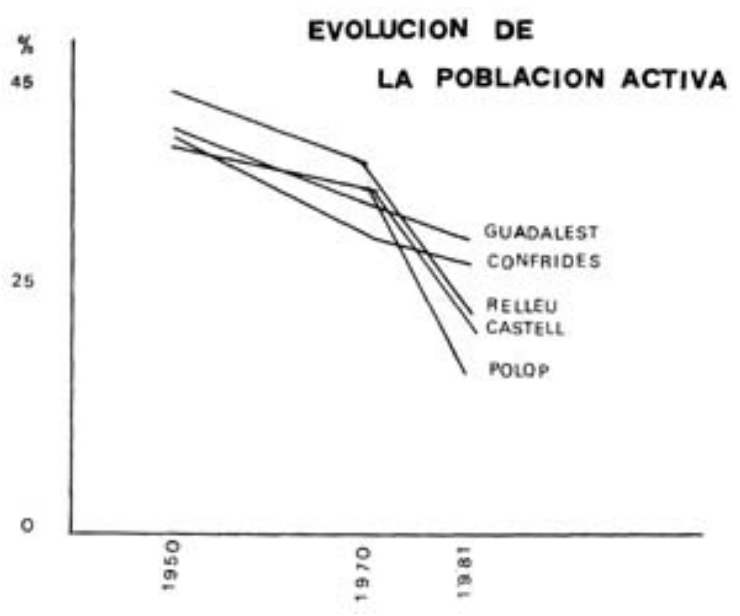

Figura 2.

te «clandestina», ya que es conocida por todos, por los organismos oficiales y por los habitantes del pueblo en cuestión.

En las pirámides de población que aparecen en este trabajo es fácilmente comprobable esta actividad, ya que si existe, no aparecen censadas estas actividades industriales y el lector creería que en la actualidad sólo viven de la agricultura. Es el caso de pueblos como Relleu.

\section{Cuadro 1}

ESTRUCTURA PROFESIONAL EN 1950

\begin{tabular}{|c|c|c|c|c|c|c|c|c|c|}
\hline & P.T. & P.A. & $\%$ & I & $\%$ & II & $\%$ & III & $\%$ \\
\hline Castell de C. & 977 & 437 & $44^{\prime} 7$ & 383 & $87^{\prime} 6$ & 11 & $2 ' 5$ & 43 & $99^{\prime} 8$ \\
\hline Confrides & 774 & 306 & $39^{\prime} 5$ & 289 & $94^{\prime} 4$ & 5 & $1^{\prime} 6$ & 12 & $3^{\prime} 9$ \\
\hline Guadalest & 333 & 138 & $41^{\prime} 4$ & 132 & $95^{\prime} 6$ & - & - & 6 & $4^{\prime} 3$ \\
\hline Polop & 1.396 & - & - & - & - & & & - & \\
\hline Relleu & 1.813 & 718 & $39^{\prime} 6$ & 616 & $85^{\prime} 7$ & 46 & 64 & 56 & $7 ' 7$ \\
\hline
\end{tabular}

FUENTE: Elaboración propia a partir del Censo de 1950. 

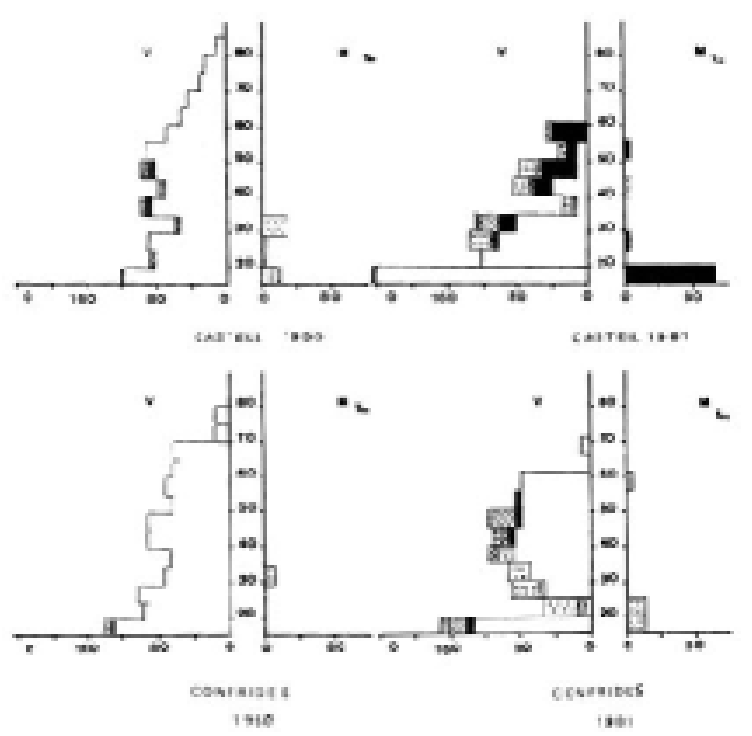

$\square \square \mathbb{Z Z : 2}$

a. $r$ e. re. a. a

Figura 3.

Cuadro II

ESTRUCTURA PROFESIONAL EN 1970

\begin{tabular}{|c|c|c|c|c|c|c|c|c|c|}
\hline & P.T. & P.A. & $\%$ & $\mathbf{I}$ & $\%$ & II & $\%$ & III & $\%$ \\
\hline Castell de C. & 797 & 296 & $37^{\prime} 2$ & 252 & $85^{\prime} 2$ & 23 & $7 ' 3$ & 21 & $6 \cdot 5$ \\
\hline Confrides & 499 & 151 & $30^{\prime} 2$ & 125 & $82^{\prime} 4$ & 15 & $10^{\prime} 3$ & 11 & $7 ' 3$ \\
\hline Guadalest & 218 & 80 & $37^{\prime} 6$ & 67 & $84^{\prime} 4$ & 5 & $6 ' 3$ & 8 & $9^{\prime} 3$ \\
\hline Polop & 1.554 & 565 & $36^{\prime} 4$ & 232 & $41^{\prime} 2$ & 184 & $32^{\prime} 6$ & 149 & $26^{\prime} 2$ \\
\hline Relleu & 1.307 & 457 & $35^{\prime} 0$ & 388 & $85^{\prime} 0$ & 45 & $9^{\prime} 9$ & 24 & $5^{\prime} 1$ \\
\hline
\end{tabular}

FUENTE: Nota 1. 

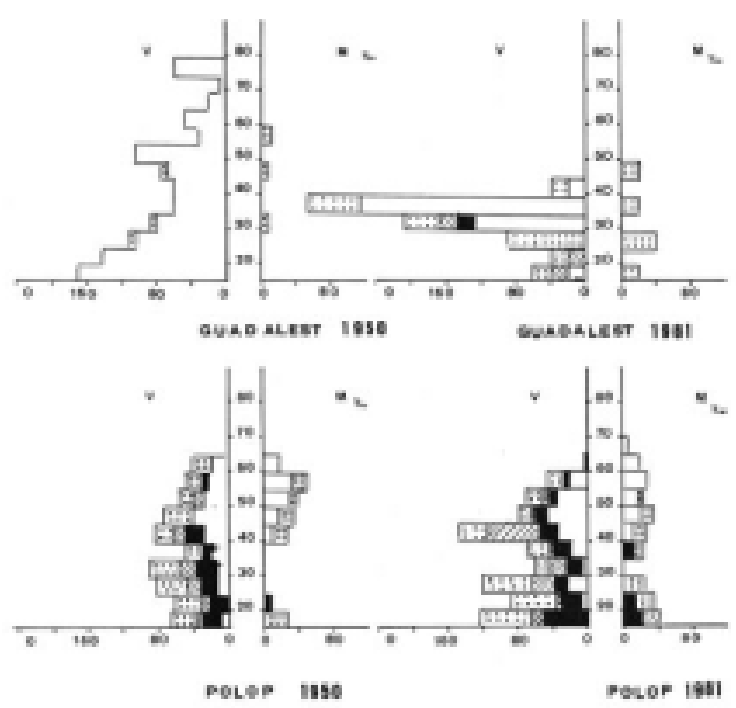

Figura 4.

Cuadro III

ESTRUCTURA PROFESIONAL EN 1981

\begin{tabular}{lrrlrlrrrr}
\hline & P.T. & P.A. & \% & \multicolumn{1}{l}{ I } & \% & \multicolumn{1}{l}{ II } & \multicolumn{1}{c}{$\%$} & \multicolumn{1}{l|}{ III } & \% \\
\hline Castell de C. & 695 & 151 & $21^{\prime} 7$ & 93 & $61^{\prime} 5$ & 39 & $25^{\prime} 8$ & 19 & $12^{\prime} 5$ \\
Confrides & 368 & 102 & $27^{\prime} 7$ & 71 & $69^{\prime} 6$ & 10 & $99^{\prime} 8$ & 21 & $20^{\prime} 5$ \\
Guadalest & 158 & 46 & $29^{\prime} 1$ & 22 & $47^{\prime} 8$ & 5 & $10^{\prime} 8$ & 19 & $41^{\prime} 3$ \\
Polop & 1.766 & 299 & $16^{\prime} 9$ & 88 & $29^{\prime} 4$ & 94 & $31^{\prime} 4$ & 117 & $39^{\prime} 1$ \\
Relleu & 1.009 & 235 & $23^{\prime} 2$ & 176 & $74^{\prime} 8$ & 20 & $8^{\prime} 5$ & 39 & $16^{\prime} 5$ \\
\hline
\end{tabular}

FUENTE: Elaboración propia a partir del Censo de 1981. 


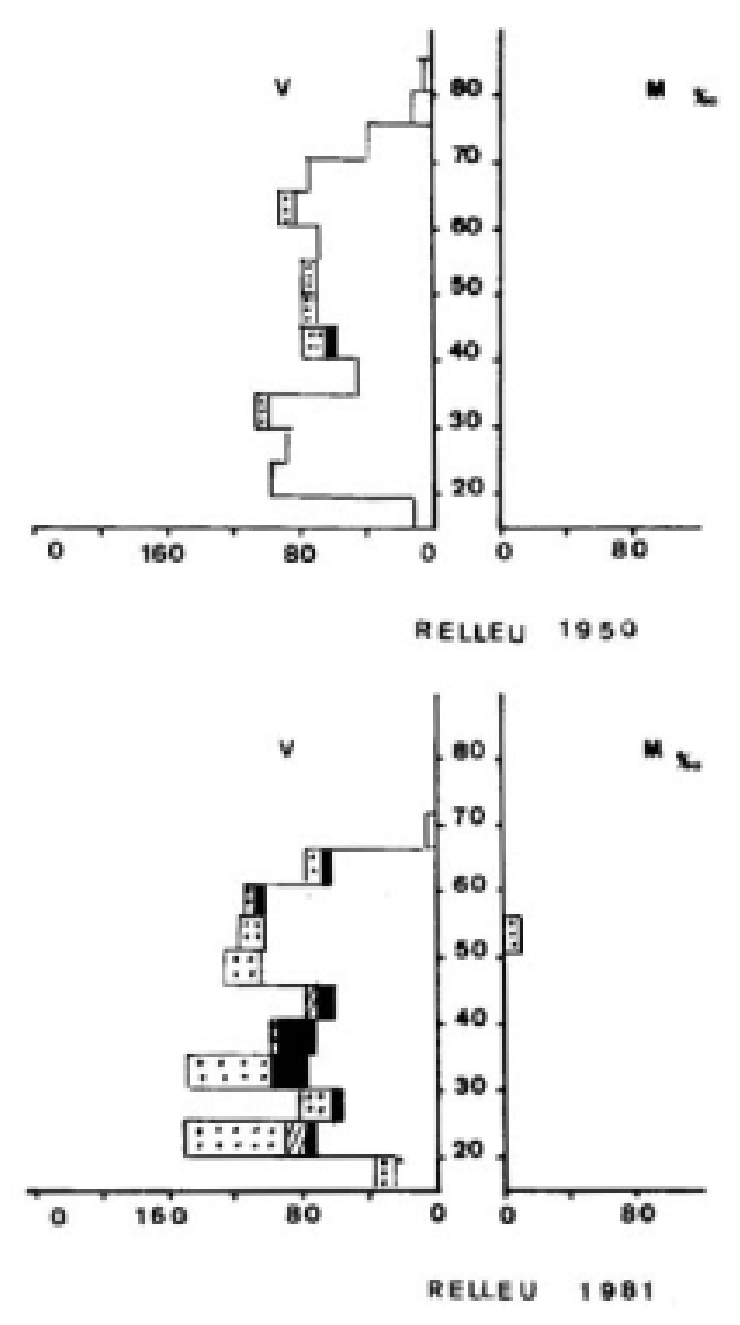

Figura 5.

\section{Estructura profesional}

Un rasgo que destaca sobre los demás es la disminución de la población activa municipal entre 1950 y 1981. De cifras superiores a un $40 \%$ en 1950 (Castell de Castells y Guadalest), y con porcentajes de 39\% en Confrides y 39'6 en Relleu, se pasa a cifras tan bajas como un $16^{\prime} 9 \%$ en Polop en $1981^{2}$. El resto de los pueblos presenta valores por encima del $20 \%$ en todos ellos: Castell de Castells, Confrides, Guadalest y Relleu.

Analizando separadamente los sectores de actividad se constata la pérdida generalizada de los activos agrarios en todos los pueblos. Hecho que se patentiza con mayor efectividad desde $1970^{3}$. Así, los porcentajes del sector primario en 1970 no habían experimentado tanta diferencia con respecto a los de 1950, moviéndose entre valores del $85 \%$ en todos los pueblos, excepto Polop.

En 1981 se pierden 23'7\% puntos en Castell de Castells, 12'8\% en Confrides, 36'6\% en Guadalest, 11'8\% en Polop y 10'2\% en Relleu. 


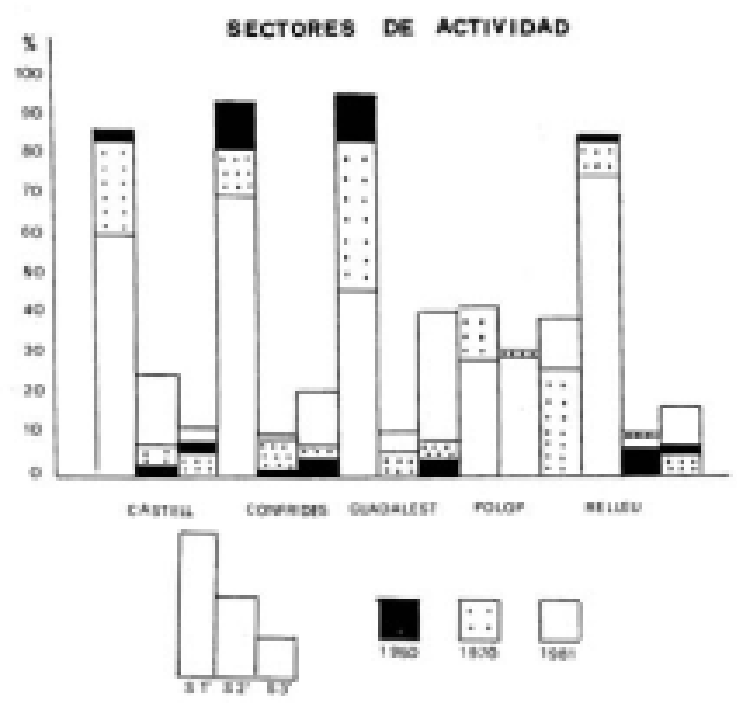

Figura 6.

De todas formas y a pesar de esta brusca pérdida de población laboral dedicada a las tareas del campo, aún hoy se pueden clasificar como pueblos agrarios, según el porcentaje de su población activa, los siguientes: Castell de Castells, Confrides y Relleu.

En Guadalest están bastante aproximadas las cifras del sector primario (47'8\%) y del terciario o servicios $\left(41^{\prime} 3 \%\right)$.

El caso de Polop es diferente. Este municipio no es agrario sino terciario (39'1\%), con una buena proporción de trabajadores dedicados a la industria (laboratorio fotográfico e industria de la construcción, 31'4\%).

El ramo de la construcción es el que proporciona un mayor número de activos dentro del secundario, debido a la proximidad del área turística de Benidorm y las posibilidades de empleo que encuentran los vecinos de Polop en esta zona. Una gran parte de esta población que ha cambiado de actividad sigue residiendo en sus municipios de origen, los 
radios de acción de 10 ó $15 \mathrm{~km}$. de distancia con respecto al lugar de trabajo suponen una emigración pendular diaria sobre todo entre los municipios limítrofes del área de Benidorm.

El secundario tiene escaso valor en la comarca. Dentro de este sector se individualiza con personalidad propia el ramo de la construcción. El turismo del área costera de municipios como Benidorm, Altea y Villajoyosa se ha traducido en una demanda de brazos para la construcción de viviendas residenciales y de alojamientos hoteleros. El resto de las actividades industriales son puramente artesanas, destacando las industrias de consumo, carpinterías, mecánica en general, etc. En 1950 se ha pasado de porcentajes insignificantes (2'5\% en Castell de Castells, 1'6\% en Confrides y 6'4\% en Relleu) a valores próximos al 10\% en 1970 para todos los casos, excepto Polop, que acusa el boom demográfico de Benidorm y las áreas costeras en los años setenta, intensificándose el fenómeno de la construcción.

En 1981 destaca el ejemplo de Castell de Castells, donde el porcentaje industrial alcanza el 25 '8\% debido a la actividad que proporciona la instalación de fábricas de bolsos y marroquinería, lo que supone que una cuarta parte de la población activa municipal se dedique a estos menesteres.

Polop se mantiene estacionario en este censo de 1981 (se acusa la crisis del 73 y sus consecuencias en años posteriores).

Los demás pueblos no presentan ninguna importancia.

El terciario es el gran protagonista de la década de los años ochenta. Se ha incrementado de forma extraordinaria debido sobre todo al fenómeno turístico. Este sector presenta una amplia diversidad de funciones, desde la burocrática, propia de empleados y administrativos del Ayuntamiento, el comercio, banca, hostelería y profesiones liberales.

En el censo de 1950 ningún pueblo superaba el 10\% de su población activa dedicada a este sector.

En 1970 los porcentajes seguían manteniéndose prácticamente en idénticas proporciones, destacando Polop, que contaba con un 26'2\%.

En 1981 las cifras se habían duplicado, triplicado o incluso más en todos los pueblos estudiados. Paradójicamente donde menos había aumentado era en Polop.

En resumen, se observa en estos treinta años un cambio de funciones en las ramas de actividad bastante significativo. Los municipios estudiados ofrecen el ejemplo de numerosos pueblos de nuestra provincia, donde ha descendido en cifras absolutas la población activa dedicada al sector primario en más de la mitad. Los pueblos que aún siguen siendo agrícolas, por encima de otros sectores económicos como la industria o los servicios, han perdido más de la mitad de su población, hallándose ésta envejecida. Se asiste a un trasvase de funciones económicas, el primario deja de ser el sector principal en beneficio del terciario o de la industria.

La industria es poco representativa en estos pequeños municipios, tratándose generalmente de actividades artesanales. El terciario es, sin lugar a dudas, el que va cobrando un auge tremendo en los últimos años, ligado sobre todo a la creación de puestos de trabajo en el sector de la banca, hostelería y ocio en un área que goza de la privilegiada situación que supone ser vecinos espacialmente del gran Benidorm.

\section{Notas, bibliografía y fuentes}

1. QUEREDA SALA, J:, La Marina. Estudio de geografía regional, Excma. Diputación Provincial de Alicante, 1978. 
2. Se ha constatado en Polop que existen relaciones laborales que no se ajustan a las normas legales vigentes en la sociedad actual (no se encuentran censados como asalariados ni como población activa).

3. QUEREDA SALA, J., Ibíd., dice que se revela predominante el sector primario. El agricultor caracteriza profesionalmente la población comarcal (pp. 325 y ss.).

Censos de población de 1950 y 1981. 